\title{
Design of Constructive Controller of Nonlinear System Based on Polynomial Function Growth Condition and Its Application in Deep Subsea Energy Mining and Production Control System
}

\author{
Longchuan Guo $\mathbb{C D}^{1,2}$ Chuanping Zhou, ${ }^{1,2}$ Xiaoqing Tian, ${ }^{1}$ Huawei Ji, ${ }^{1}$ and Yudong Peng ${ }^{1}$ \\ ${ }^{1}$ School of Mechanical Engineering, Hangzhou Dianzi University, Hangzhou 310018, China \\ ${ }^{2}$ Department of Computer Information Engineering, National Technical University of Ukraine, Kyiv 03056, Ukraine \\ Correspondence should be addressed to Longchuan Guo; glc1988@126.com
}

Received 14 August 2021; Accepted 16 September 2021; Published 7 October 2021

Academic Editor: Nan Zhang

Copyright (c) 2021 Longchuan Guo et al. This is an open access article distributed under the Creative Commons Attribution License, which permits unrestricted use, distribution, and reproduction in any medium, provided the original work is properly cited.

\begin{abstract}
This paper mainly studies the output feedback control problem of the stochastic nonlinear system based on loose growth conditions and applies the research results to the valve control system of underwater oil and gas pipelines, which can improve the speed and stability of the equipment system. First, the concept of randomness is introduced to study the actual tracking control problem of output feedback of stochastic nonlinear systems, remove the original harsher growth conditions, make it meet the more general polynomial function growth conditions, and propose a combination of static and dynamic output feedback practices. The design of the tracking controller makes all the states of the system meet boundedness and ensures that the tracking error of the system converges to a small neighborhood of zero. Second, the system is extended to the parameter-uncertain system, and the output feedback tracking controller with complete dynamic gain is constructed by proving the boundedness of the system state and gain. Further, the time-delay factor is introduced, and the nonlinear term of the system satisfies the more relaxed power growth condition, combined with the inverse method to cleverly construct a set of Lyapunov functions and obtain the output controller to ensure that the system is asymptotically probabilistic in the global scope. Stability. Finally, through the ocean library in the Simulation $X$ simulation software, the controller design results are imported into the underwater electro-hydraulic actuator model to verify the effectiveness of the controller design.
\end{abstract}

\section{Introduction}

Stability and robustness are two important basic concepts in system and control science. However, there is a difference between the two. Stability describes the ability of the system to maintain relative to changes in the initial state, while robustness describes the ability of the system to maintain relative to changes in the external environment or the system itself. The robustness analysis and control law design of the control system are the main aspects of the research problems in robust control theory [1-3]. Robustness analysis mainly studies the stability and dynamic performance of the control system in the presence of uncertainty or external interference. The main research of control law design is the need to design an effective control law to make the system with uncertainty and external interference more robust. After more than 30 years of development, robust control theory has achieved a series of results, and it has become one of the most active fields in the research content of control theory [4-6].

In the 1990s, the authors in [3] first proposed a sliding mode variable structure control method, and the authors in $[5,6]$ further developed sliding mode variable structure control theory. In 2010, the research on sliding mode variable structure in [7] attracted widespread attention from the control community. Since then, scholars have begun to study sliding mode variable structure control in depth and formed a complete system, and sliding mode variable structure control has also begun. It is applied to complex systems such as discrete systems, uncertain 
systems, time-delay systems, nonlinear systems, and switching systems. Sliding mode variable structure control is a control strategy of variable structure control systems. Its fundamental difference from conventional control lies in the discontinuity of control, that is, a switching characteristic that makes the "structure" of the system change over time. Sliding mode variable structure control can force the system to move along a prescribed state trajectory under certain characteristic conditions. Since the sliding mode can be designed and has nothing to do with the parameters and disturbances of the system, all systems in sliding mode motion have good robustness.

In [8], the author introduced the development history of sliding mode variable structure, the definition and mathematical expression of sliding mode, and so on. Combined with the introduction in the article, we focus on the introduction of discrete-time system sliding mode variable structure control. For discrete systems, sliding mode variable structure control cannot produce an ideal sliding mode but can only produce a quasi-sliding mode [9]. Discrete sliding mode variable structure control mainly discusses three basic issues: the existence, accessibility, and stability of sliding modes.

Based on this, uncertain nonlinear time-delay systems are an important class of nonlinear systems. At present, there have been many mature research results for the control design theory of uncertain nonlinear time-delay systems or single uncertain nonlinear systems under strong constraints. However, in practical engineering problems, most uncertain nonlinear time-delay systems need to meet relatively loose constraints, and some engineering conditions are even unconstrained. It is particularly worth noting that in the field of deep-sea oil and gas development, due to the influence of different components in pipeline, the opening process model of underwater electro-hydraulic valve satisfies the Hölder constraint condition; at the same time, the electronic integrated modules of underwater electro-hydraulic systems generally use chaotic circuits containing piecewise linear memristors (piecewise linearization of nonlinearities is also one of the important methods for studying nonlinear systems) [10-12]. Based on the above analysis, the system characteristics of the two engineering problems include nonlinearity, uncertainty, and piecewise linearity, and the good control of the underwater electro-hydraulic valve actuator and its electronic module determines the overall speed, stability, and realtime and reliability of oil and gas pipeline operation, which are related to the overall safety and reliability of offshore oil and gas production.

Combining the above research and analysis, the main innovative contributions of this paper are as follows:

(1) Different from the research object in [13-16], the state equation of the control object we studied in this chapter contains both a time-delay term and an uncertain parameter, including a nonlinear function term of the state and time delay. Among them, the uncertainty includes the uncertainty of the state and time delay, the uncertainty of the disturbance, and the uncertainty of the Hölder condition nonlinear function.

(2) From the description in [17], we can see that many robust controller designs for uncertain nonlinear time-delay systems are based on the known state of the system. A state observer is established to obtain the state estimate, and further a robust controller is designed.

(3) Simulation $X$ optimization tool is an important and effective method in optimal control. The optimal control performance mentioned in [18] can be obtained by solving LMIs. In this paper, by establishing a new Lyapunov-Krasovskii functional, the Lyapunov stability theorem and Simulation $X$ method will be applied to the design of robust controllers for nonlinear systems based on observers.

(4) The nonlinear term in the nonlinear system is assumed to satisfy the Hölder condition described in $[19,20]$.

(5) This chapter introduces and models an electrohydraulic valve position control system and applies the designed observer and controller to the system. The model includes uncertain parameter disturbances, hydraulic transmission time lag, and nonuniformity that meets Hölder's conditions, linear friction, and so on.

\section{Description of Valve Control System Model of Offshore Gas Transportation Pipeline}

Electro-hydraulic control systems are widely used in aerospace systems, vehicle systems, artillery launch base control and oil extraction, and so on and have a wide range of applications in actual production and processes. In this section, we consider an application to the offshore oil and gas production process. The electro-hydraulic valve position control system [21-24] mainly focuses on the output practical tracking controller design for a class of complex stochastic nonlinear systems with unknown control coefficients. As the technical accumulation of paper [24], a solution is provided for increasing the nonlinear constraint conditions for this paper, and it is just suitable for underwater control valve systems.

The electro-hydraulic composite valve position control system studied in this section has been applied in actual offshore engineering such as oil and gas exploration and production, especially in the field of deep-sea oil and gas development. Its core hub part-underwater hydraulic actuators, due to the influence of different component substances in pipelines, the system presents uncertain and nonlinear characteristics, and for underwater hydraulic actuators, fast response time and good tracking performance are two extremely important indicators in actual oil and gas production applications because they determine the speed, stability, real-time, and reliability of the operation of the entire oil and gas pipeline and are related to the overall safety and reliability of offshore oil and gas production. 
The electro-hydraulic compound valve position control system is composed of a water equipment part and an underwater equipment part (as shown in Figure 1). The water part includes liquid supply unit, hydraulic station, other power units, and other control units; the underwater part includes electromagnetic reversing valve, hydraulic actuator, and the electronic control unit that controls the electromagnetic reversing valve; the inside of the electronic control unit is the controller logic design.

In actual marine engineering applications, in order to ensure the stability, reliability, and safety of the system, the response speed of the valve position control needs to be stable, accurate, and timely; during the valve position opening/closing process, it is also affected by the hydraulic driving force, spring restoring force, and the force generated by the fluid medium inside the oil and gas pipeline during the opening process of the actuator.

Combined with the existing research results, the electrohydraulic compound valve position control system model in the process of valve position opening is considered as follows:

$$
\begin{aligned}
p\left(S-S_{1}\right)+\rho g H S_{1}= & m \frac{d^{2} \tau}{\mathrm{d} t^{2}}+\left(\rho g H+\frac{128 \mu l_{p} Q}{\pi d_{p}^{4}}\right)\left(S-S_{2}\right) \\
& +k\left(L_{0}+\tau\right)+f\left(\tau, \frac{\mathrm{d} \tau}{\mathrm{d} t}\right)+w(t), \\
Q_{h}= & v\left(S-S_{1}\right)\left(2 * \frac{\left(P_{1}-P_{2}\right)}{\rho_{h}}\right) .
\end{aligned}
$$

According to the characteristics of offshore oil and gas transportation pipelines, we generalize the pipeline transportation model to obtain a broad mathematical model with unknown control directions as follows:

$$
\begin{aligned}
d x_{i} & =g_{i}(t, x) x_{i+1} \mathrm{~d} t+f_{i}(t, x, u) \mathrm{d} \omega, \quad(i=1, \ldots, n-1) \\
d x_{n} & =g_{n}(t, x) u \mathrm{~d} t+f_{n}(t, u, x) \mathrm{d} \omega \\
y & =x_{1}-y_{r}(t)
\end{aligned}
$$

where $x=\left(x_{1}, \ldots, x_{n}\right)^{T} \in R^{n}, u \in R$, and $y \in R$ are the states, input, and output of system; corresponding to this, $y_{r}$ is a given unmeasurable output trajectory. Here, we introduce a stochastic process for the system: $\omega$ is an $m$-dimensional standard Wiener process defined on the complete probability space $(\Omega, \Gamma, \mathrm{P})$ with $\Omega$ being a sample space, $\Gamma$ being a filtration, and $\mathrm{P}$ being a probability measure. $g_{i}(t, x)(i=1, \ldots, n)$ is the control coefficient and is bounded; furthermore, the control coefficient is unknown, and the first variable is continuous, and the second variable is local Lipschitz. Nonlinear term $f_{i}: R^{+} \times R \times R^{n} \longrightarrow$ $R^{m}, i=1, \ldots, n$, is continuous for $t$ and locally Lipschitz in $(u, x)$.

At present, there have been some results related to the stability analysis of Hölder nonlinear systems, but there are no relevant results in the research on the control problems of uncertain nonlinear time-delay systems that meet such conditions, and they also include uncertainties and time delays. Nonlinear systems with complex factors and other complex factors have certain practical application research value; further, it is necessary to consider the design of state observers when state variables are unmeasurable and the design of robust controllers based on state observers. In order to achieve the control objectives, the system and reference signals are required to satisfy following assumptions.

Assumption 1. There exists positive integer $p$ and known positive constant $c_{0}$ such that the following inequality holds:

$$
\left|f_{i}(t, x, u)\right| \leq c_{0}\left(1+\left|x_{1}\right|^{p}\right)\left(\left|x_{1}\right|+\cdots+\left|x_{i}\right|\right)+c_{0}, \quad i=1, \ldots, n .
$$

Based on this assumption, system (2) is dominated by an output polynomial function growth rate system.

Assumption 2. The symbol of $g_{i}(t, x),(i=1, \ldots, n)$ is defined as the control direction of system (3), and control direction in this system is unknown. $g_{i}(t, x)$ takes the value on the unknown interval $\left[\xi_{i}, \zeta_{i}\right]$ in which $\xi_{i}$ and $\zeta_{i}$ are the upper and lower bounds of the determined constant interval.

Assumption 3. The reference output trajectory $y_{r}$ of system (3) is continuously differentiable and satisfies the following inequality:

$$
\max \left(\left|y_{r}\right|,\left|\dot{y}_{r}\right|\right) \leq c_{1}
$$

where $c_{1}$ is the known positive constant.

According to the characteristics and constraints of the above system, it leads to the objective of this paper: for any constant $\delta>0$, all the states of stochastic nonlinear system (3) are well defined and bounded. In addition, there exists a finite time $T>0$ such that for any $t>T$, it can get

$$
|y(t)|=\left|x_{1}(t)-y_{r}(t)\right| \leq \delta .
$$

Remark 1. There have been some research studies on this type of system, and some results have been obtained. For example, in $[23,25,26]$, the random impulse control problem of the system under Hölder conditions is analyzed. Among the problems studied in this chapter, compared with previous studies, the described nonlinear system conditions have a stronger scope of application. On the basis of the nonlinear system analysis method, considering the problem of robust sliding mode controller design for piecewise linear discrete time-delay systems with state lag and uncertain parameters, applying sliding mode control theory to piecewise linear time-delay systems can make the system state move along a given "sliding mode" motion trajectory and apply the sliding mode variable structure control theory to the electro-hydraulic compound valve position control system, which has practical significance in the control theory and application of the piecewise linear system. 


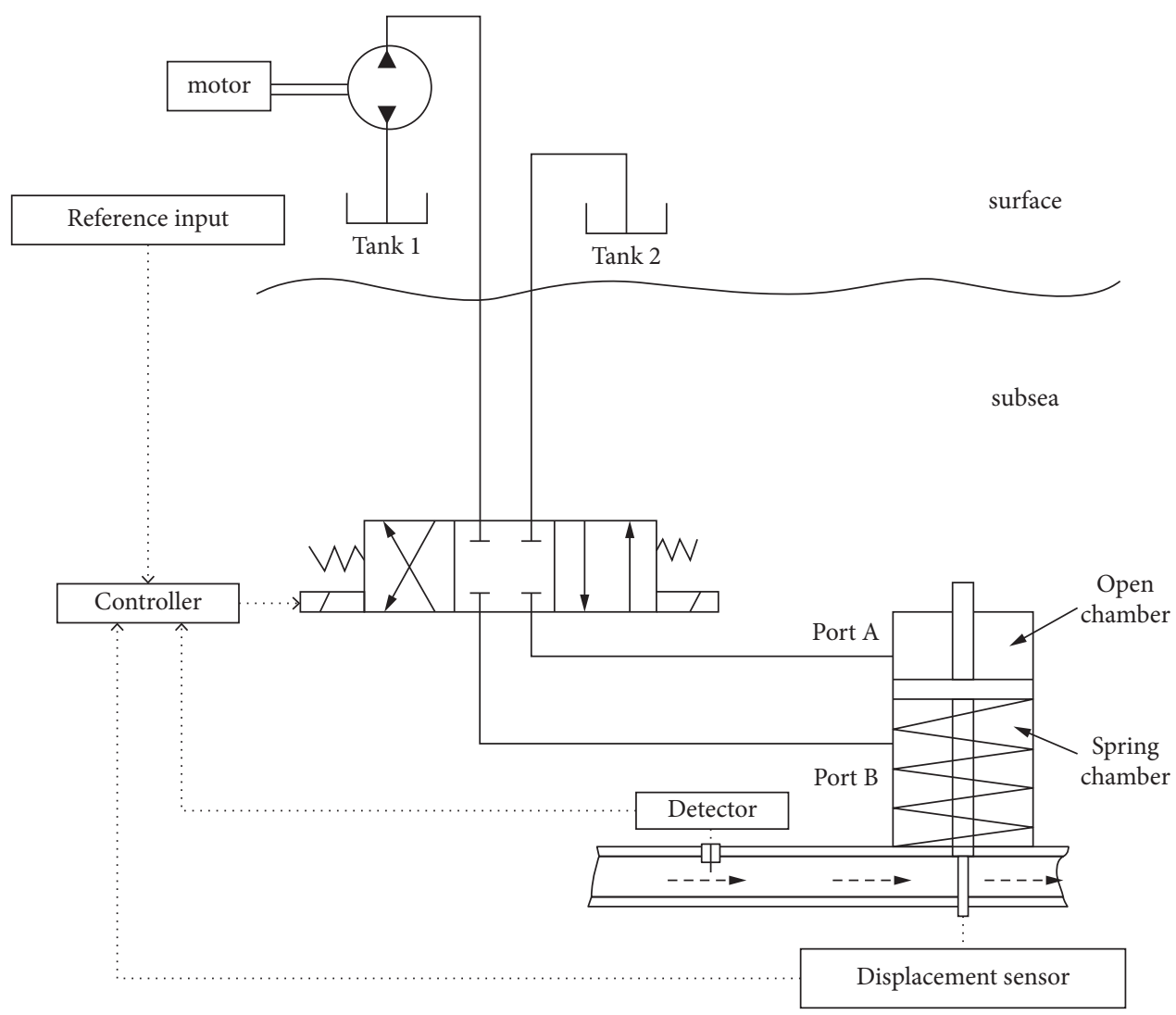

FIgURE 1: Underwater electro-hydraulic composite valve position control system.

\section{Complex Tracking Control Design}

According to the description of the stochastic nonlinear system with unknown control coefficients (1) and Assumptions $1-3$, we can get

$$
\left|f_{i}(t, x, u)\right| \leq c\left(1+|y|^{p}\right)\left(\left|x_{1}\right|+\cdots+\left|x_{i}\right|\right)+c
$$

where $c=c_{0} \max \left(1+2^{p-1} c_{1}^{p}, 2^{p-1}\right)$ is a known constant.

For the sake of convenience of calculation and formula derivation, the following simple states transformation is introduced and then it can obtain the updated stochastic nonlinear system:

$$
\begin{aligned}
d z_{i} & =g_{i}(t, z) z_{i+1} \mathrm{~d} t+F_{i}(t, u, z) \mathrm{d} \omega, \\
d z_{n} & =g_{n}(t, z) u \mathrm{~d} t+F_{n}(t, u, z) \mathrm{d} \omega, \\
y & =z_{1} .
\end{aligned}
$$

Based on the simplicity of formula derivation, especially introducing state transitions:

$$
\begin{aligned}
z_{1} & =x_{1}-y_{r}, \\
z_{i} & =x_{i}, \quad(i=2, \ldots, n),
\end{aligned}
$$

definition $F_{i}(t, u, z)$ is as follows:

$$
F_{i}(t, u, z)=g_{i} f_{i}\left(t, u, z_{1}+y_{r}, z_{2}, \cdots, z_{n}\right) .
$$

States observer of system (3) is established as follows:

$$
\begin{aligned}
& \dot{\vec{z}}_{i}=\widehat{z}_{i+1}+K^{i} l_{i}\left(z_{1}-\widehat{z}_{1}\right), \quad(i=1, \ldots, n-1), \\
& \dot{\vec{z}}_{n}=u+K^{n} l_{n}\left(z_{1}-\widehat{z}_{1}\right),
\end{aligned}
$$

where $l_{i}>0(i=1, \ldots, n)$ is the coefficient of the Hurwitz polynomial with $s^{n}+l_{1} s^{n-1}+\cdots+l_{n-1} s+l_{n} . K=R T(t)$ is a high-order gain term which consists of a constant $R$ and a variable $T(t)$ as follows:

$$
\dot{T}=-\alpha_{1} T^{2}+\alpha_{2}\left(1+|y|^{p}\right)^{2} T, \quad T(0)=1 .
$$

Define the system error states as $e_{i}=z_{i}-\widehat{z}_{i}$ $(i=1, \ldots, n)$, and then by (6)-(9), the error system can be obtained:

$$
\begin{aligned}
& d e_{i}=\|\xi\| \cdot\|\zeta\| e_{i+1} \mathrm{~d} t-K^{i} l_{i} e_{1} \mathrm{~d} t+F_{i}(t, u, z) \mathrm{d} \omega \\
& d e_{n}=-\|\xi\| \cdot\|\zeta\| K^{n} l_{n} e_{1} \mathrm{~d} t+F_{n}(t, u, z) \mathrm{d} \omega .
\end{aligned}
$$

Remark 2. On the basis of designing observers for nonlinear systems, scholars have launched the observer-based controller design. Among them, the controller design of the observer combining the Lyapunov stability theorem and the LMIs method has achieved certain research results. In [23], the author studied the robust controller design of the state observer for uncertain time-delay systems. From the above research results, it can be obtained that robust control has a wide range of applications, and it also reflects the degree of attention paid to the research of uncertain nonlinear systems with time delays. However, on the other 
hand, most of the research results mentioned at present are the study of time-delay nonlinear systems, state-uncertain nonlinear systems, or both time-delay characteristics and parameter-uncertain nonlinear systems. There are relatively few research studies on observers of time delay, state uncertainty, and time-delay uncertainty, as well as observers of nonlinear discrete systems that include state variables and nonlinear functions of time-delay variables and robust control based on observers.

Transformation of estimated states $\widehat{z}_{i}$ and error states $e_{i}$ is introduced to further simplify subsequent controller design issues:

$$
\begin{aligned}
\varepsilon_{i} & =\frac{e_{i}}{K^{b+i-1}}, \quad(i=1, \ldots, n), \\
\tau_{i} & =\frac{\widehat{z}_{i}}{K^{b+i-1}}, \quad(i=1, \ldots, n),
\end{aligned}
$$

where $0 \leq b \leq(1 / p)\|\xi\| \zeta\|\|$ is the known constant. Further, by (12), systems (7)-(9) can be converted into the following:

$$
\begin{aligned}
\mathrm{d} \varepsilon & =K L\|\xi\|\|\zeta\| \varepsilon d t-\frac{\dot{K}}{K} C_{b} \varepsilon \mathrm{d} t+G(z, K) \mathrm{d} \omega, \\
\dot{\tau} & =K L^{T} \tau\|\xi\|\|\zeta\|+K l_{1}-\frac{\dot{K}}{K} C_{b} \tau,
\end{aligned}
$$

where

$$
\begin{aligned}
L & =\left(\begin{array}{cccc}
-l_{1} & 1 & \cdots & 0 \\
\vdots & \vdots & \ddots & \vdots \\
-l_{n-1} & 0 & \cdots & 1 \\
-l_{n} & 0 & \cdots & 0
\end{array}\right), \\
L^{T} & =\left(\begin{array}{cccc}
0 & 1 & \cdots & 0 \\
\vdots & \vdots & \ddots & \vdots \\
0 & 0 & \cdots & 1 \\
-l_{1} & -l_{2} & \cdots & -l_{n}
\end{array}\right), \\
C_{b} & =\operatorname{diag}(b, b+1, \ldots, b+n-1), \\
G(z, K) & =\left(\frac{F_{1}}{K^{b}}, \ldots, \frac{F_{n}}{K^{b+n-1}}\right)^{T} .
\end{aligned}
$$

According to the system description and definition in the second section, it is concluded that the closed-loop system has a unique solution in interval $\left[0, t_{T}\right)$. Moreover, if $T$ is maximum value of $t_{f}$, then $0<T \leq \infty$.

Next, we need to discuss the boundedness of the various states and parameter variables of the system. Appropriate parameters $r_{1}, r_{2}, r_{3}$, and $r_{4}$ are chosen such that positive definite matrix $P, Q$ and matrix $L, L^{T}, C_{b}$ satisfy the following relation:

$$
\begin{aligned}
& r_{1} I_{n} \leq C_{b} P+P C_{b} \leq r_{2} I_{n}, \\
& r_{3} I_{n} \leq C_{b} Q+Q C_{b} \leq r_{4} I_{n} .
\end{aligned}
$$

Define the following Ito Lyapunov function $V(\varepsilon, \tau)=\varepsilon^{T} P \varepsilon+\tau^{T} Q \tau$, and then it can get the trajectory of (16) along the Ito differentiation for system (13) as follows:

$$
\begin{aligned}
L V= & -K|\varepsilon|^{2}\|\zeta\|\|\xi\|+2 \varepsilon^{T} P G-\frac{\dot{K}}{K} \varepsilon^{T}\left(C_{b} P+P C_{b}\right) \varepsilon-2 K|\tau|^{2}\|\zeta\|\|\xi\| \\
& +2 K \tau^{T} \varepsilon_{1} h-\|\zeta\|\|\xi\| \frac{\dot{K}}{K} \tau^{T}\left(C_{b} Q+Q C_{b}\right) \tau-\frac{\dot{K}}{K} \varepsilon^{T}\left(C_{b} P+P C_{b}\right) \varepsilon \\
& \leq r_{4} \alpha_{1} B\|\zeta\|\|\xi\||\tau|^{2}-r_{3} \alpha_{2}\left(1+|y|^{p}\right)^{2}|\tau|^{2},
\end{aligned}
$$

where

$$
|G|=\left(\left(\frac{F_{1}}{K_{b}}\right)^{2}+\cdots+\left(\frac{F_{n}}{K^{b+n-1}}\right)^{2}\right)^{1 / 2},
$$

where (17) can be magnified to

$$
\begin{aligned}
& (\|P\|+\|G\|)\left(c\left(1+|y|^{p}\right)\left(\sqrt{n}(|\varepsilon|+|\tau|)+c_{1}\right)+c+c_{1}\right)^{2}, \\
& \quad \leq(\|P\|+\|G\|) \cdot\left(n c^{2}\left(1+|y|^{p}\right)^{2}\left(|\varepsilon|^{2}+|\tau|^{2}\right)\right. \\
& \left.\quad+4 c^{2}\|\zeta\|\|\xi\| c_{1}^{2}+2\left(c+c_{1}\right)^{2}\right) .
\end{aligned}
$$

By inequality scaling principle, parameter $\alpha_{2}$ can be chosen as follows:

$$
\alpha_{2} \geq \frac{\|\zeta\|\|\xi\| c\|P\|\left(1+c_{1}\right)-n c^{2}(\|P\|+\|Q\|)}{r_{3}} .
$$

Since $T \geq 1$, parameter $R$ needs to satisfy the following relationship:

$$
R \geq \frac{\|\zeta\|\|\xi\|\|P\|\left(n c+c+c_{1}\right)+r_{2} \alpha_{1}}{1-\|\zeta\|\|\xi\||l|^{2}} \geq 1 .
$$

According to the above for the choice of parameters, then (22) can be translated into the following:

$$
\begin{aligned}
L V \leq & -\frac{R}{\max \left(\lambda_{\max }(P)+\lambda_{\max }(Q)\right)} \cdot V+\|\zeta\|\|\xi\|\|P\|\left(c c_{1}+c+c_{1}\right) \\
& +\left(c^{2}\left(1+|y|^{p}\|\zeta\|\|\xi\|\right)^{2} c_{1}^{2}+\left(c+c_{1}\right)^{2}\right)(\|P\|+\|Q\|) .
\end{aligned}
$$

By using other variables instead of the above derivation results, we can get

$$
\begin{aligned}
& E_{1}=\frac{R}{\max \left(\lambda_{\max }(P)+\lambda_{\max }(Q)\right)} \\
& E_{2}=\|\zeta\|\|\xi\|\|P\|\left(c c_{1}+c+c_{1}\right)+\left(\left(1+|y|^{p}\right)^{2} c_{1}^{2}+1\right) .
\end{aligned}
$$

Since the closed-loop system has a unique solution in interval $[0, \infty)$, by $(21)-(23)$, we can obtain 


$$
V(\varepsilon(t), \tau(t)) \leq V(\varepsilon(0), \tau(0)) e^{-\left(E_{1} / A\right) t}+\frac{E_{2}}{E_{1}}, \quad t \in[0, T),
$$

such that states $\varepsilon(t)$ and $\tau(t)$ of stochastic nonlinear system (7) $-(9)$ and (13) are well defined and bounded on $[0, T)$.

Correspondingly, by $y=z_{1}=\left(\varepsilon_{1}+\tau_{1}\right) K^{b}$ and $|y| \leq$ $(R T)^{b}\left(\left|\varepsilon_{1}\right|+\left|\tau_{1}\right|\right)$, we can get $|y| \leq(A B)^{b} E_{3}$. According to the boundedness of $\varepsilon$ and $\tau$ on $[0, T)$, combined with the above obtained $|y| \leq(R T)^{b} E_{3}$ and $b \in(0,(1 / p)\|\zeta\|\|\xi\|)$, we can obtain

$$
T(t) \leq \frac{\alpha_{2}^{2}\|\zeta\|\|\xi\|\left(1+E_{3}^{2 p} \sqrt{R}\right)^{2}}{\alpha_{1}^{2}} .
$$

Through the above analysis, we can prove that the system gain $T(t)$ is well defined and bounded on $[0, T)$ with $T=\infty$.

Through the above analysis, it can be concluded that the system is essentially bounded. Then, we give the theorem of output feedback practical tracking controller.

Theorem 1. By choosing appropriate parameters $a_{i}, l_{i}, \alpha_{1}$, and $\alpha_{2}$, the following output feedback practical tracking controller can be designed:

$$
u=-\left(K^{n} a_{1} \widehat{z}_{1}+K^{n-1} a_{2} \widehat{z}_{2}+\cdots+K a_{n} \widehat{z}_{n}\right),
$$

where $K$ is defined by (10) and $a_{i}>0$ is the coefficient of Hurwitz polynomial. Since the closed-loop stochastic nonlinear system satisfies locally Lipschitz condition, it is concluded that the closed-loop system has a unique solution in interval $\left[0, t_{T}\right)$. Moreover, if $T$ is maximum value of $t_{f}$, then $0<T \leq \infty$. There exists a time $T=T_{1}$ such that

$$
V(\varepsilon(t), \tau(t)) \leq \frac{2 E_{2}}{E_{1}}, \quad t \geq T=T_{1} .
$$

According to (19)-(25), we can conclude that

$$
\lambda_{\min }(P)|\varepsilon|^{2}+\lambda_{\min }(Q)|\tau|^{2} \leq V(\varepsilon(t), \tau(t)) .
$$

Further squaring formula (28), we can get

$$
\varepsilon_{1}^{2}(t)+\tau_{1}^{2}(t) \leq \frac{E_{4}}{R} .
$$

By using $|y| \leq(A B)^{b} E_{3}$ and (27)-(29), we can get

$$
|y| \leq A^{b} B^{b}\|\xi\| \zeta\|\|\left(\left|\varepsilon_{1}(t)\right|+\left|\tau_{1}(t)\right|\right) \leq \sqrt{\frac{\|\xi\| \zeta\|\| E_{4}}{A}} .
$$

Simultaneously, combined with (30), we can get further

$$
y^{2}(t) \leq \frac{\|\xi\| \zeta\|\| E_{4}}{A}
$$

Remark 3. In the control design program, the design problem of the controller is transformed into a parameter construction problem by introducing the appropriate coordinate transformation, and the forward push and saturation control design method based on the iterative program is not used. The controller gain obtained by the iterative design method may become too small during multiple iterations. The obtained controller is theoretically effective, but it is difficult to achieve in engineering practice, and when the dimensionality of the system is relatively high, the multiple iteration procedures will greatly increase the difficulty and complexity of the design. The controller designed in this project has a simple form and moderate dynamic/static gain strength, so it is easier to apply to engineering practice. In this article, we take the underwater electro-hydraulic valve system as an example and apply the robust controller designed above to verify the effectiveness of the controller.

\section{Offshore Gas Pipeline Valve Transportation Model Example}

The underwater electro-hydraulic compound valve position control system is used in actual marine engineering such as oil and gas exploration and production, especially in the field of deep-sea oil and gas development. Its core hub partunderwater hydraulic actuator is affected by different components in the pipeline. The system presents uncertainties and nonlinear characteristics. The electro-hydraulic composite valve position control system is composed of aquatic equipment part and an underwater equipment part (as shown in Figure 1). The water part includes liquid supply unit, hydraulic station, other power units, and other control units; underwater part includes electromagnetic the reversing valve, hydraulic actuator, and the electronic control unit for controlling the electromagnetic reversing valve, and the inside of the electronic control unit is the controller logic design.

Combining existing research results $[27,28]$, the model of the electro-hydraulic composite valve position control system during the valve position opening process is obtained as follows:

$$
\begin{aligned}
p\left(S-S_{1}\right)+\rho g H S_{1}= & m \frac{d^{2} \tau}{\mathrm{d} t^{2}}+\left(\rho g H+\frac{128 \mu l_{p} \mathrm{Q}}{\pi d_{p}^{4}}\right) \\
& \cdot\left(S-S_{2}\right)+k\left(L_{0}+\tau\right)+f\left(\tau, \frac{\mathrm{d} \tau}{\mathrm{d} t}\right)+w(t), \\
Q_{h}= & v\left(S-S_{1}\right)\left(2 * \frac{\left(P_{1}-P_{2}\right)}{\rho_{h}}\right) .
\end{aligned}
$$

In the above formula, the physical meaning of each parameter is shown in Table 1.

Due to the pressure instability of the oil and gas medium and the sand and other components in the formation, it will cause certain interference in the opening process of the electro-hydraulic composite valve position, and the viscosity coefficient of the control fluid changes with temperature, and there are factors such as vibration in the hydraulic system. The resistance during the opening process has the characteristics of satisfying the Hölder condition. According 
TABle 1: Variable description.

\begin{tabular}{lccc}
\hline Variable & Meaning & Variable & Meaning \\
\hline$p$ & Valve opening cavity supply pressure & $H$ & Working water depth \\
$\tau$ & Valve position & $L_{0}$ & Spring precompression displacement \\
$S$ & Piston area & $S_{1}$ & Piston rod area \\
$S_{2}$ & Stem area & $m$ & Piston rod quality \\
$\rho$ & Sea water density & $\mu$ & Control fluid viscosity \\
$k$ & Spring coefficient of elasticity & $g$ & Acceleration of gravity \\
$d_{p}$ & Inner diameter of pilot hole & $f$ & $Q_{p}$ \\
$Q_{h}$ & Control fluid flow & $P_{2}$ & Resistance during opening of the actuator \\
$P_{1}$ & Control fluid inlet pressure & $\rho_{h}$ & Flow rate of fluid medium in pipeline \\
$v$ & Control fluid flow rate & $l_{p}$ & Control fluid outlet pressure \\
$\sigma$ & Pressure coefficient & $\eta$ & Control liquid density \\
$v_{p}$ & Flow rate of fluid medium in pipeline & Control the length of the liquid guide hole \\
& & Valve opening
\end{tabular}

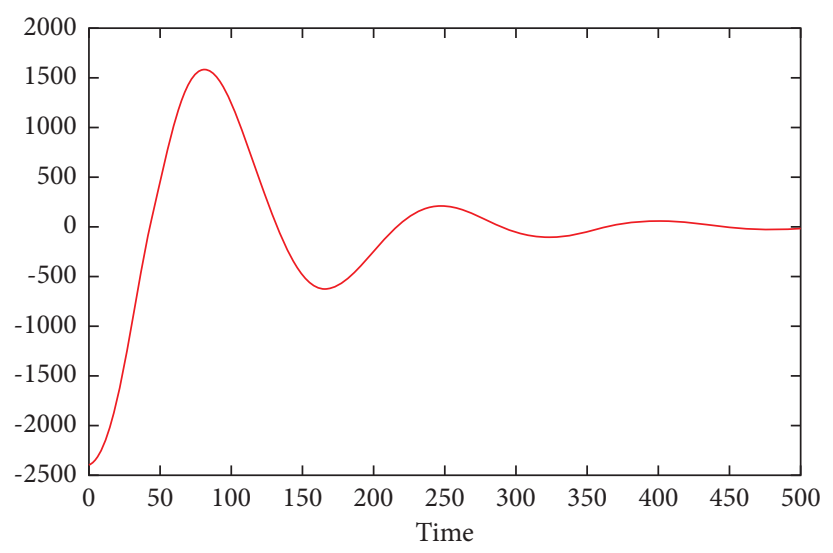

Figure 2: The trajectory of system output $y=x_{1}-y_{r}$.

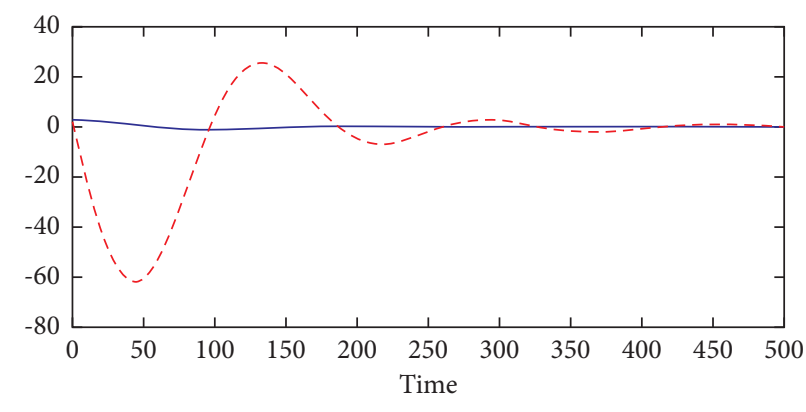

$-\mathrm{x} 1$

$---\mathrm{x} 2$

FIgURE 3: The trajectory of system state $x_{1}$ and $x_{2}$.

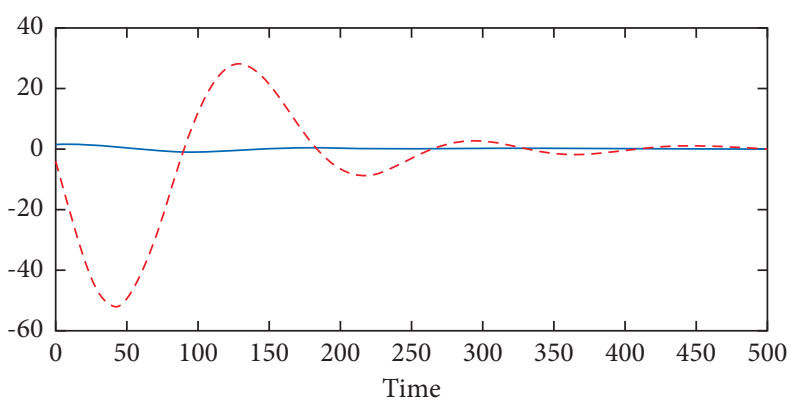

$\begin{aligned} \text { - } & \text { x1 observer } \\ -- \text { - } & \text { x2 observer }\end{aligned}$

FIgURE 4: The trajectory of system observer state $x_{1}$ and $x_{2}$. 
to the decomposition of the actual model and the comparative study, we can completely believe that the control system model of the marine pipeline valve is completely matched with the theoretical control law and control model obtained above:

$$
\begin{aligned}
x_{1} & =\tau, \\
x_{2} & =\frac{\mathrm{d} \tau}{\mathrm{d} t} \\
\mathrm{~d} x_{1} & =x_{2} \mathrm{~d} t+\mathrm{d} x_{1}^{p(x)} \sin x_{2} \mathrm{~d} \omega, \\
d x_{2} & =u \mathrm{~d} t+\mathrm{d}\left(x_{1}^{p(x)}+x_{2}^{(x)}\right) \mathrm{d} \omega, \\
y & =x_{1} .
\end{aligned}
$$

Here, we set the initial value as

$$
\left[x_{1}(0), x_{2}(0), \widehat{z}_{1}(0), \widehat{z}_{2}(0)\right]=[2.5,1,1.5,-5] \text {. }
$$

Figures 2-4 show the response curve of the closed-loop system 1 . Observed by the simulation diagram, the state of the two-dimensional systems (1)-(3) is bounded and gradually converges, and the system can also be observed. The output tracking error gradually converges and finally adjusts to near zero. The simulation results verify the effectiveness of the designed output feedback actual tracking controller.

\section{Concluding and Future Prospects}

In this paper, we study the design of the system observer under the condition that the state of the system is unmeasurable and the design of the system controller based on the observer. The system has uncertain parameter disturbances, time-delay terms, and nonlinear terms. By introducing the error between the observation value and the actual value, the error equation and the closed-loop loop of the observer-based control system are combined, using the Lyapunov method for stability analysis, and finally the relevant theorems obtained from the stability analysis are combined to design the observer and the controller. The Simulation $X$ is used to solve the robust controller under the given parameters; finally, the electro-hydraulic compound valve position control model is established, and the valve position of its actuator through the controller designed is analyzed in this paper. In this paper, we study the design of system observer and controller when state variables are unmeasurable based on the stability theorem. The nonlinear system studied includes uncertain parameter disturbances and time-delay terms.

However, in the actual research process, there is a class of nonlinear systems that can be piecewise linearized. The system model is a piecewise linear model; that is, a nonlinear system is composed of finite or infinite linear subsystems [29-32]. Piecewise linear systems are widely used in practice. In the life and production process, the piecewise linear system is also an important approximation method for the nonlinear system. The nonlinear system can be described by the piecewise linear system. Therefore, it is necessary to study the control problem of the piecewise linear system.

\section{Data Availability}

The data generated or analyzed during this research are included in this paper.

\section{Conflicts of Interest}

The author declares that there are no conflicts of interest regarding the publication of this paper.

\section{Acknowledgments}

This work was supported by the National Natural Science Foundation of China under Grant 61807010, Key R\&D Program of Zhejiang Province under Grant 2021C03013, Zhejiang Provincial Natural Science Foundation of China under Grant LZ21E050002, Fundamental Research Funds for Provincial Universities of Zhejiang under Grants GK199900299012-026 and GK219909299001-309.

\section{References}

[1] Y. Mizuta and T. Shimomura, “'Sobolev's inequality for Riesz potentials with variable exponent satisfying a log-Hölder condition at infinity," Journal of Mathematical Analysis and Applications, vol. 311, no. 1, pp. 268-288, 2005.

[2] J.-Q. Li, Y.-S. Zhang, C.-M. Ji, A.-J. Wang, and J. R. Lund, "Large-scale hydropower system optimization using dynamic programming and object-oriented programming: the case of the Northeast China Power Grid," Water Science and Technology, vol. 68, no. 11, pp. 2458-2467, 2013.

[3] Q. Liu and Z. Liang, "Global stabilisation of a class of feedforward systems with distributed delays," IET Control Theory \& Applications, vol. 9, no. 1, pp. 140-146, 2015.

[4] R. Raoufi, H. J. Marquez, and A. S. I. Zinober, " $\mathscr{H}$ Eo sliding mode observers for uncertain nonlinear Lipschitz systems with fault estimation synthesis," International Journal of Robust and Nonlinear Control, vol. 20, no. 16, pp. 1785-1801, 2010.

[5] M. Abbaszadeh and H. J. Marquez, "LMI optimization approach to robustHoobserver design and static output feedback stabilization for discrete-time nonlinear uncertain systems $\mathrm{H}$ infinity observer design and static output feedback stabilization for discrete-time nonlinear uncertain systems," International Journal of Robust and Nonlinear Control, vol. 19, no. 3, pp. 313-340, 2009.

[6] X. Chen, X. Zhang, C. Zhang, and L. Chang, "Global asymptotic stabilization for input-delay chained nonholonomic systems via the static gain approach," Journal of the Franklin Institute, vol. 355, no. 9, pp. 3895-3910, 2018.

[7] D. Soudry, D. Di Castro, A. Gal, A. Kolodny, and S. Kvatinsky, "Memristor-based multilayer neural networks with online gradient descent training," IEEE transactions on neural networks and learning systems, vol. 26, no. 10, pp. 2408-2421, 2015.

[8] P. Kokotovic and M. Arcak, "Constructive nonlinear control: a historical perspective," Automatica, vol. 37, no. 5, pp. 637-662, 2001.

[9] T. Chen and J. Huang, "A small gain approach to global stabilization of nonlinear feedforward systems with input 
unmodeled dynamics," Automatica, vol. 46, no. 6, pp. 1028-1034, 2010.

[10] M. Krstic, "Feedback linearizability and explicit integrator forwarding controllers for classes of feedforward systems," IEEE Transactions on Automatic Control, vol. 49, no. 10, pp. 1668-1682, 2004.

[11] L. Long and J. Zhao, "Global stabilization for a class of switched nonlinear feedforward systems," Systems \& Control Letters, vol. 60, no. 9, pp. 734-738, 2011.

[12] X. Ye, "Adaptive stabilization of time-delay feedforward nonlinear systems," Automatica, vol. 47, no. 5, pp. 950-955, 2011.

[13] H. Ye, "Saturated delayed controls for feedforward nonlinear systems," IEEE Transactions on Automatic Control, vol. 59, no. 6, pp. 1646-1653, 2014.

[14] B. Bao, F. Hu, and Z. Liu, "Mapping equivalent approach to analysis and realization of memristor-based dynamical circuit," Chinese Physics B, vol. 23, no. 7, pp. 303-310, 2014.

[15] M. Pai, "RBF-based discrete sliding mode control for robust tracking of uncertain time-delay systems with input nonlinearity," Complexity, vol. 21, 2015.

[16] L. Praly and Z. P. Jiang, "Linear output feedback with dynamic high gain for nonlinear systems," Systems \& Control Letters, vol. 53, no. 2, pp. 107-116, 2004.

[17] M.-S. Koo, H.-L. Choi, and J.-T. Lim, "Global regulation of a class of uncertain nonlinear systems by switching adaptive controller," IEEE Transactions on Automatic Control, vol. 55, no. 12, pp. 2822-2827, 2010.

[18] X. Zhang and Y. Lin, "Global adaptive stabilisation of feedforward systems by smooth output feedback," IET Control Theory \& Applications, vol. 6, no. 13, pp. 2134-2141, 2012.

[19] X. Zhang, H. Gao, and C. Zhang, "Global asymptotic stabilization of feedforward nonlinear systems with a delay in the input," International Journal of Systems Science, vol. 37, no. 3, pp. 141-148, 2006.

[20] H.-W. Jo, H.-L. Choi, and J.-T. Lim, "Observer based output feedback regulation of a class of feedforward nonlinear systems with uncertain input and state delays using adaptive gain," Systems \& Control Letters, vol. 71, pp. 44-53, 2014.

[21] L.-C. Guo, X. Zuo, J.-W. Liu, and H.-Q. Liang, "Outputfeedback control of a class of stochastic nonlinear systems with power growth conditions," International Journal of Control, Automation and Systems, vol. 12, no. 2, pp. 274-282, 2014.

[22] L.-C. Guo and J.-W. Liu, "Improved backstepping control for stochastic high-order nonlinear time delay system with a constructive mechanical system," Transactions of the Institute of Measurement and Control, vol. 40, no. 15, pp. 4115-4124, 2018.

[23] X. Chen and X. Zhang, "Output-feedback control strategies of lower-triangular nonlinear nonholonomic systems in any prescribed finite time," International Journal of Robust and Nonlinear Control, vol. 29, no. 4, pp. 904-918, 2019.

[24] L. Guo and X. Fang, "Bounded analysis and practical tracking control of complex stochastic nonlinear systems with unknown control coefficients," Complexity, vol. 2, Article ID 1502065, 2021.

[25] X. Chen, X. Zhang, and C. “Zhang, L. Chang, A time-varying high-gain approach to feedback regulation of uncertain timevarying nonholonomic systems," ISA Transactions, vol. 98, pp. 110-122, 2020.

[26] X. Chen, X. Zhang, and Q. Liu, "Prescribed-time decentralized regulation of uncertain nonlinear multi-agent systems via output feedback," Systems \& Control Letters, vol. 137, Article ID 104640, 2020.

[27] A. Benabdallah, M. Hammami, and J. Kallel, "Robust stability of uncertain piecewise-linear systems: LMI approach," Nonlinear Dynamics, vol. 63, no. 1, pp. 183-192, 2011.

[28] C. Briat, "Stability analysis and control of a class of LPV systems with piecewise constant parameters," Systems \& Control Letters, vol. 82, pp. 10-17, 2015.

[29] P. Krishnamurthy and F. Khorrami, "Feedforward systems with ISS appended dynamics: adaptive output-feedback stabilization and disturbance attenuation," IEEE Transactions on Automatic Control, vol. 53, no. 1, pp. 405-412, 2008.

[30] X. Zhang, Q. Liu, L. Baron, and E.-K. Boukas, "Feedback stabilization for high order feedforward nonlinear time-delay systems," Automatica, vol. 47, no. 5, pp. 962-967, 2011.

[31] X. Zhang, L. Baron, Q. Liu, and E.-K. Boukas, "Design of stabilizing controllers with a dynamic gain for feedforward nonlinear time-delay systems," IEEE Transactions on Automatic Control, vol. 56, no. 3, pp. 692-697, 2011.

[32] W. Zha, J. Zhai, and S. Fei, "Global output feedback control for a class of high-order feedforward nonlinear systems with input delay," ISA Transactions, vol. 52, no. 4, pp. 494-500, 2013.

[33] Z. Liang and Q. Liu, "Design of stabilizing controllers of upper triangular nonlinear time-delay systems," Systems \& Control Letters, vol. 75, pp. 1-7, 2015. 